\title{
A MONOTONE ITERATIVE METHOD FOR BOUNDARY VALUE PROBLEMS OF PARAMETRIC DIFFERENTIAL EQUATIONS
}

\author{
XINZHI LIU ${ }^{1}$ \\ University of Waterloo \\ Department of Applied Mathematics \\ Waterloo, Ontario, Canada N2L 3G1 \\ FARZANA A. MCRAE \\ Catholic University of America \\ Department of Mathematics \\ Washington, DC 20057 USA
}

(Received June, 1997; Revised June, 2000)

This paper studies boundary value problems for parametric differential equations. By using the method of upper and lower solutions, monotone sequences are constructed and proved to converge to the extremal solutions of the boundary value problem.

Key words: Upper and Lower Solutions, Monotone Iteration, Boundary Value Problems, Parametric Differential Equations.

AMS subject classifications: $34 \mathrm{~B} 15,34 \mathrm{~K} 10$.

\section{Introduction}

Many problems in physical chemistry and physics, describing the exothermic and isothermal chemical reactions, the steady-state temperature distributions, the oscillation of a mass attached by two springs, lead to differential equations with a parameter [5]. Existence, uniqueness and approximate solutions of problems with a parameter have been discussed in [1] where several other references may be found.

Recently, the method of upper and lower solutions and monotone iterative technique was initiated for boundary value problems with a parameter [2]. In this paper, we shall extend the work of [2], under weaker assumptions in the general set up of the standard work [3] so that further advances can be made for such problems.

\footnotetext{
${ }^{1}$ Research partially supported by NSERC-Canada.
} 


\section{Main Results}

Consider the parametric equation

$$
\left\{\begin{array}{c}
x^{\prime}=f(t, x, \lambda) \\
0=g(x, \lambda) \\
x(0)=A
\end{array}\right.
$$

where $f \in C\left[J \times R^{2}, R\right], g \in C[\Omega \times R, R]$, where $J=[0, T], \Omega=\{u: u \in C[J, R]\}$.

Definition 2.1: A pair $(v, \alpha), v \in C^{1}[J, R]$ and $\alpha \in R$, is said to be a lower solution of $(2.1)$ if

$$
\left\{\begin{array}{c}
v^{\prime} \leq f(t, v, \alpha) \\
0 \leq g(v, \alpha) \\
v(0) \leq A
\end{array}\right.
$$

An upper solution of $(\omega, \beta)$ of $(2.1)$ can be defined similarly by reversing the above inequalities.

Theorem 2.1: Assume that

(i) $\quad v, w \in C^{1}[J, R], \alpha, \beta \in R,(v, \alpha)$ and $(w, \beta)$ are lower solutions of (2.1), respectively, such that $\alpha \leq \beta$ and $v(t) \leq w(t), t \in J$;

(ii) $f \in C\left[J \times R^{2}, R\right], f(t, x, \lambda)$ is nondecreasing in $\lambda$ and for $M \geq 0$ such that

$$
f(t, x, \lambda)-f(t, \tilde{x}, \lambda) \geq-M(x, \tilde{x})
$$

whenever $v(t) \leq \tilde{x} \leq x \leq w(t), t \in J$ and $\alpha \leq \lambda \leq \beta$;

(iii) $g \in C[\Omega \times R, R], g(\bar{x}, \lambda)$ is nondecreasing in $\bar{x}$ and for $N \geq 0$ such that

$$
g(x, \lambda)-g(x, \tilde{\lambda}) \geq-N(\lambda-\tilde{\lambda})
$$

whenever $v(t) \leq x \leq w(t), t \in J, \alpha \leq \tilde{\lambda} \leq \lambda \leq \beta$.

Then, there exists monotone sequences $\left\{v_{n}, \alpha_{n}\right\},\left\{w_{n}, \beta_{n}\right\}$ which converge monotonically to minimal and maximal solutions $(\rho, k),(\gamma, l)$ of $(2.1)$, respectively, i.e.,

$$
\begin{gathered}
v \leq v_{1} \leq \ldots \leq v_{n} \leq \rho \leq \gamma \leq w_{n} \leq \ldots \leq w_{1} \leq w \\
\alpha \leq \alpha_{1} \leq \ldots \leq \alpha_{n} \leq k \leq l \leq \beta_{n} \leq \ldots \leq \beta_{1} \leq \beta, \quad \forall n .
\end{gathered}
$$

Proof: For any $\eta \in\langle v, w\rangle$, where

$$
\langle v, w\rangle=\left\{x \in C\left[J, R^{n}\right]: v(t) \leq x(t) \leq w(t), \quad t \in J\right.
$$

and $e \in[\alpha, \beta]$, we consider the linear parametric equation

$$
\left\{\begin{array}{c}
x^{\prime}+M x=\sigma(t, e), \quad t \in J \\
0=g(\eta, e)-N(\lambda-e), \\
x(0)=A
\end{array}\right.
$$

where $\sigma(t, e)=f(t, \eta(t), e)+M \eta(t)$. 
It is not difficult to see that problem $(2.3)$ possesses a unique solution $(x(t), \lambda)$ for any given pair $(\eta(t), e)$. Let $\left(v_{1}, \alpha_{1}\right),\left(w_{1}, \beta_{1}\right)$ be the solutions of $(2.3)$ corresponding $(\eta, e)=(v, \alpha)$ and $(\eta, e)=(w, \beta)$, respectively.

Let $p=\alpha-\alpha_{1}$. We then get

$$
0=g(v, \alpha)-N\left(\alpha_{1}-\alpha\right) \geq-N\left(\alpha_{1}-\alpha\right)=N p,
$$

which implies $p \leq 0$ and hence $\alpha \leq \alpha_{1}$. Similarly, we can show $\beta_{1} \leq \beta$. Now let $p=\alpha_{1}-\beta_{1}$. By condition (iii), we get

$$
\begin{gathered}
0=g(v, \alpha)-N\left(\alpha_{1}-\alpha\right)=g(v, \alpha)-g(w, \beta)-N\left(\alpha_{1}-\alpha\right)+N\left(\beta_{1}-\beta\right) \\
\leq g(w, \alpha)-g(w, \beta)-N\left(\alpha_{1}-\alpha\right)+N\left(\beta_{1}-\beta\right) \\
N(\beta-\alpha)-N\left(\alpha_{1}-\alpha\right)+N\left(\beta_{1}-\beta\right)=-N p .
\end{gathered}
$$

Hence $\alpha_{1} \leq \beta_{1}$. Thus, we get

$$
\alpha \leq \alpha_{1} \leq \beta_{1} \leq \beta
$$

Next, we shall show

$$
v(t) \leq v_{1}(t) \leq w_{1}(t) \leq w(t), \quad t \in J
$$

Let $m(t)=v(t)-v_{1}(t), t \in J$. Then

$$
\begin{gathered}
m^{\prime}=v^{\prime}(t)-v_{1}^{\prime}(t) \leq f(t, v(t), \alpha)-f(t, v(t), \alpha)-M\left(v(t)-v_{1}(t)\right) \\
=-M m(t)
\end{gathered}
$$

and $m(0) \leq A-A=0$. Thus $m(t) \leq 0$ and $v(t) \leq v_{1}(t), t \in J$. Similarly, we can show that $w_{1}(t) \leq w(t), t \in J$. Now let $m(t)=v_{1}(t)-w_{1}(t)$. Then

$$
\begin{gathered}
m^{\prime}(t)=v_{1}^{\prime}(t)-w_{1}^{\prime}(t)=f(t, v(t), \alpha)-M\left(v_{1}(t)-v(t)\right) \\
-f(t, w(t), \beta)+M\left(w_{1}(t)-w(t)\right) \\
\leq f(t, v(t), \beta)-f(t, w(t), \beta)-M\left(v_{1}(t)-w_{1}(t)\right)+M(v(t)-w(t)) \\
\leq-M m(t)
\end{gathered}
$$

$m(0)=v_{1}(0)-w_{1}(0)=0$. Thus we get $m(t) \leq 0$ and $v_{1}(t) \leq w(t), t \in J$. Hence (2.5) is proved.

Now let $\eta_{1}, \eta_{2} \in\langle v, w\rangle, e_{1}, e_{2} \in[\alpha, \beta]$ such that

$$
\eta_{1}(t) \leq \eta_{2}(t), \quad t \in J, \quad e_{1} \leq e_{2} .
$$

Let $\left(x_{1}(t), \lambda_{1}\right),\left(x_{2}(t), \lambda_{2}\right)$ be solutions of $(2.3)$ corresponding to $\left(\eta_{1}, e_{1}\right)$ and $\left(\eta_{2}, e_{2}\right)$, respectively. We are going to show 


$$
x_{1}(t) \leq x_{2}(t), t \in J \text { and } \lambda_{1} \leq \lambda_{2}
$$

Let $m(t)=x_{1}(t)-x_{2}(t), t \in J$. Then by condition $(i i)$

$$
\begin{aligned}
m^{\prime}(t) & =x_{1}^{\prime}(t)-x_{2}^{\prime}(t) \\
& =f\left(t, \eta_{1}(t), e_{1}\right)-M\left(x_{1}(t)-\eta_{1}(t)\right)-f\left(t, \eta_{2}(t), e_{2}\right)+M\left(x_{2}(t)-\eta_{2}(t)\right) \\
& \leq f\left(t, \eta_{1}(t), e_{2}\right)-f\left(t, \eta_{2}(t), e_{2}\right)-M\left(\eta_{2}(t)-\eta_{1}(t)\right)-M\left(x_{1}(t)-x_{2}(t)\right) \\
& \leq-M m(t),
\end{aligned}
$$

and $m(0)=x_{1}(0)-x_{2}(0)=0$. Thus we have $m(t) \leq 0$ and $x_{1}(x) \leq x_{2}(t)$ on $J$. Set $p=\lambda_{1}-\lambda_{2}$. Then by condition (iii)

$$
\begin{gathered}
0=g\left(\eta_{1}, e_{1}\right)-N\left(\lambda_{1}-e_{1}\right)=g\left(\eta_{1}, e_{1}\right)-g\left(\eta_{2}, e_{2}\right)-N\left(\lambda_{1}-e_{1}\right)+N\left(\lambda_{2}-e_{2}\right) \\
\leq N\left(e_{2}-e_{1}\right)-N\left(\lambda_{1}-e_{1}\right)+N\left(\lambda_{2}-e_{2}\right)=-N p
\end{gathered}
$$

which implies $\lambda_{1} \leq \lambda_{2}$. Thus (2.7) is established.

It is now easy to construct sequences $\left\{\left(v_{n}(t), \alpha_{n}\right)\right\}$ and $\left\{w_{n}(t), \beta_{n}\right\}$, where $\left(v_{n}(t), \alpha_{n}\right)$ and $\left(w_{n}(t), \beta_{n}\right)$ are solutions of (2.3) corresponding to $(\eta, e)=\left(v_{n-1}, \alpha_{n-1}\right)$ and $(\eta, e)=\left(w_{n-1}, \beta_{n-1}\right)$, respectively, with $v_{0}=v, \alpha_{0}=\alpha$, $w_{0}=w$ and $\beta_{0}=\beta$. We conclude from (2.4), (2.5) and (2.7)

$$
v_{0} \leq v_{1} \leq v_{2} \leq \ldots \leq v_{n} \leq w_{n} \leq \ldots \leq w_{2} \leq w_{1} \leq w_{0} \text { on } J
$$

and

$$
\alpha_{0} \leq \alpha_{1} \leq \alpha_{2} \leq \ldots \leq \alpha_{n} \leq \beta_{n} \leq \ldots \leq \beta_{2} \leq \beta_{1} \leq \beta_{0}
$$

It then follows from the standard arguments that the sequences $\left\{\left(v_{n}(t), \alpha_{n}\right)\right\}$, $\left\{\left(w_{n}(t), \beta_{n}\right)\right\}$ converge uniformly and monotonically to $(\rho(t), k),(\gamma(t), l)$, respectively, and $(\rho(t), k),(\gamma(t), l)$ are solutions of the parametric equation $(2.1)$.

To show that $(\rho(t), k),(\gamma(t), l)$ are extremal solutions of $(2.1)$, let $(x(t), \lambda)$ be any solution of $(2.1)$ such that

$$
v(t) \leq x(t) \leq w(t), t \in J \text { and } \alpha \leq \lambda \leq \beta
$$

Suppose that for some $n$, we have

$$
v_{n}(t) \leq x(t) \leq w_{n}(t), t \in J \text { and } \alpha_{n} \leq \lambda \leq \beta_{n}
$$

Then, setting $m(t)=v_{n+1}(t)-x(t)$, we obtain by condition $(i i)$, and $(2.8)$

$$
\begin{gathered}
m^{\prime}(t)=f\left(t, v_{n}(t), \alpha_{n}\right)-M\left(v_{n+1}(t)-v_{n}(t)\right)-f(t, x(t), \lambda) \\
\leq M\left(x(t)-v_{n}(t)\right)-M\left(v_{n+1}(t)-v_{n}(t)=-M m(t),\right.
\end{gathered}
$$

and $m(0)=0$. Thus, we get $m(t) \leq 0$ and $v_{n+1}(t) \leq x(t), t \in J$. Similarly, we can 
prove $w_{n+1}(t) \geq x(t), t \in J$.

Let $p=\alpha_{n}-\lambda$. Then, in view of condition (iii) and (2.8),

$$
\begin{gathered}
0=g\left(v_{n}, \alpha_{n}\right)-N\left(\alpha_{n+1}-\alpha_{n}\right) \leq g\left(x, \alpha_{n}\right)-N\left(\alpha_{n+1}-\alpha_{n}\right) \\
=g\left(x, \alpha_{n}\right)-g(x, \lambda)-N\left(\alpha_{n+1}-\alpha_{n}\right) \\
\leq N\left(\lambda-a_{n}\right)-N\left(\alpha_{n+1}-\alpha_{n}\right)=-N p,
\end{gathered}
$$

which implies $\alpha_{n+1} \leq \lambda$. Similarly, we can show $\lambda \leq \beta_{n+1}$. We conclude by induction that (2.8) is true for all $n$ since $v_{0}(t) \leq x(t) \leq w_{0}(t), t \in J$ and $\alpha_{0} \leq \lambda \leq \beta_{0}$. Hence, it follows that

$$
\rho(t) \leq x(t) \leq \gamma(t), \quad t \in J \text { and } k \leq \lambda \leq l
$$

by taking the limit as $n \rightarrow \infty$ and the proof is therefore complete.

\section{References}

[1] Jankowski, T., Existence, uniqueness and approximate solutions of problems with a parameter, Zeszyty Naukowe Politechniki Gdańskiej, Matematyka 16 (1993), 3-167.

[2] Jankowski, T. and Lakshmikantham, V., Monotone iterations for differential equation with a parameter, J. Appl. Math. Stoch. Anal. 10 (1997), 273-278.

[3] Ladde, G.S., Lakshmikantham, V. and Vatsala, A.S., Monotone Iterative Techniques for Nonlinear Differential Equations, Pitman, Boston 1985.

[4] Liu, X., Nonlinear boundary value problems for first order impulsive integrodifferential equations, J. Appl. Math. Simulation 2 (1989), 185-198.

[5] Na, T.Y., Computational Methods in Engineering Boundary Value Problems, Academic Press, New York 1979. 


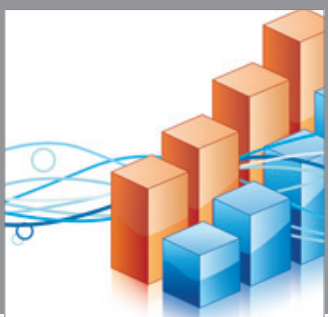

Advances in

Operations Research

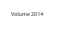

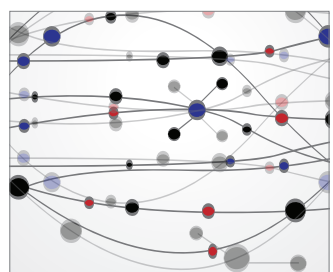

\section{The Scientific} World Journal
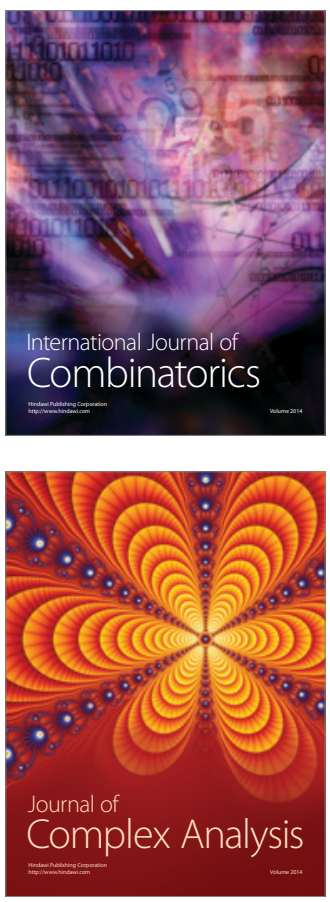

International Journal of

Mathematics and

Mathematical

Sciences
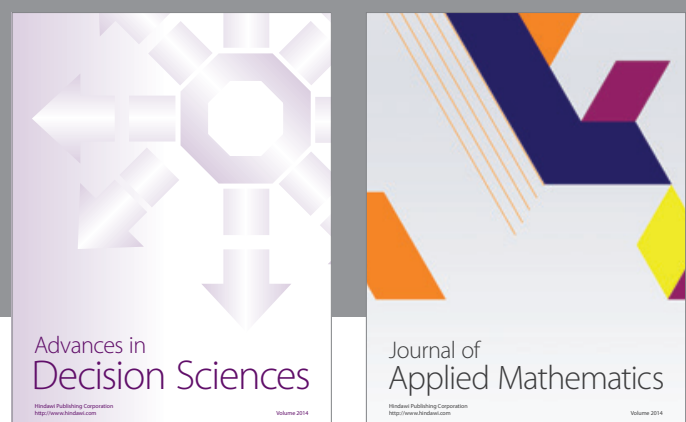

Journal of

Applied Mathematics
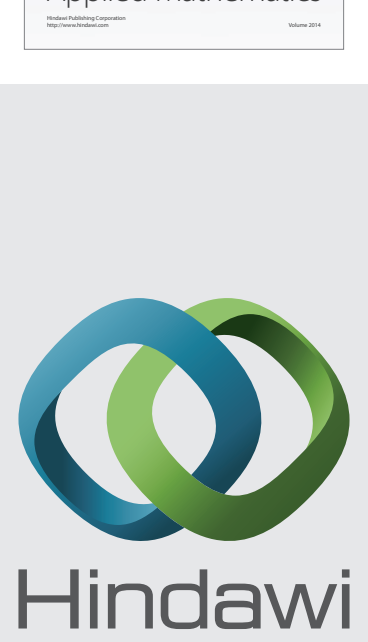

Submit your manuscripts at http://www.hindawi.com
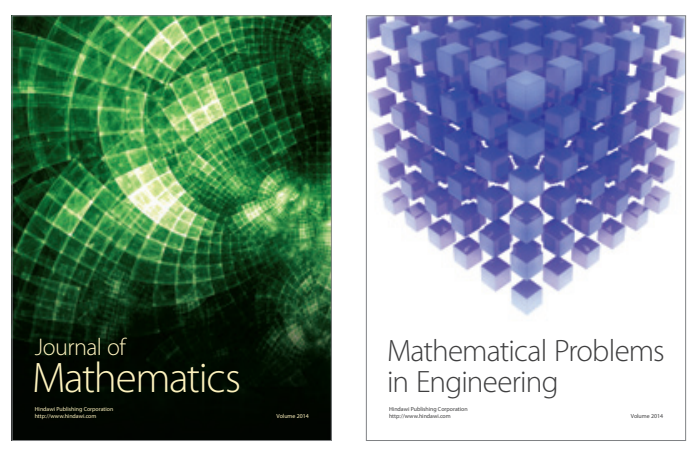

Mathematical Problems in Engineering
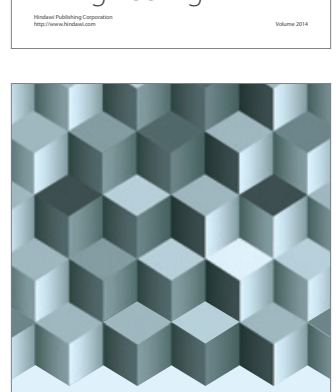

Journal of

Function Spaces
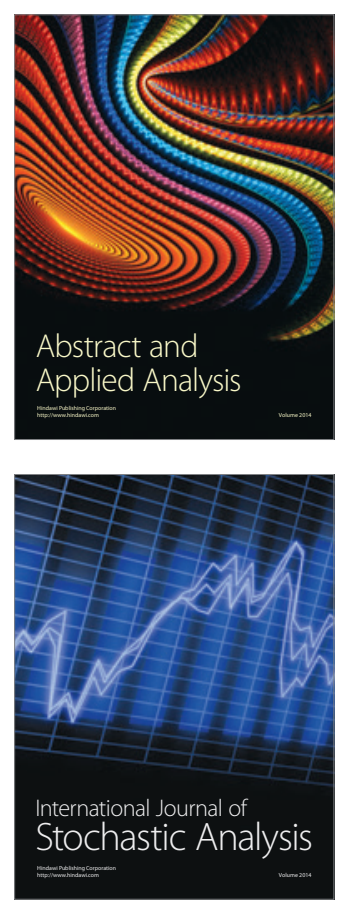

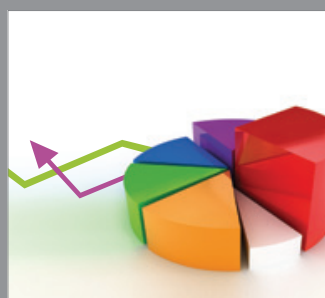

ournal of

Probability and Statistics

Promensencen
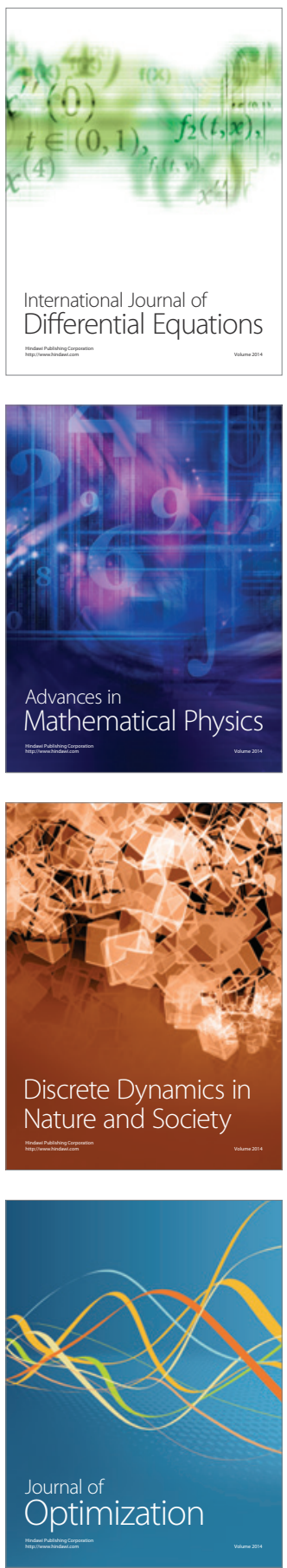